\title{
(6) OPEN ACCESS \\ Improving diagnosis and broadening the phenotypes in early-onset seizure and severe developmental delay disorders through gene panel analysis
}

\author{
Natalie Trump, ${ }^{1}$ Amy McTague, ${ }^{2,3}$ Helen Brittain, ${ }^{1}$ Apostolos Papandreou, ${ }^{2,3}$ \\ Esther Meyer, ${ }^{2,3}$ Adeline Ngoh, ${ }^{2,3}$ Rodger Palmer, ${ }^{1}$ Deborah Morrogh, ${ }^{1}$ \\ Christopher Boustred, ${ }^{1}$ Jane A Hurst, ${ }^{1}$ Lucy Jenkins, ${ }^{1}$ Manju A Kurian, ${ }^{2,3}$ \\ Richard H Scott ${ }^{1,4}$
}

\section{- Additional material is published online only. To view please visit the journal online (http://dx.doi.org/10.1136/ jmedgenet-2015-103263)}

For numbered affiliations see end of article.

\section{Correspondence to} Dr Richard H Scott, North East Thames Regional Genetics Service, Great Ormond Street Hospital, London WC1N 3JH, UK; richard.scott@gosh.nhs.uk

Received 11 May 2015 Revised 28 October 2015 Accepted 22 November 2015 Published Online First 18 March 2016

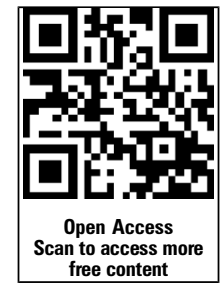

\section{ABSTRACT \\ Background We sought to investigate the diagnostic yield and mutation spectrum in previously reported genes for early-onset epilepsy and disorders of severe developmental delay. \\ Methods In 400 patients with these disorders with no known underlying aetiology and no major structural brain anomaly, we analysed 46 genes using a combination of targeted sequencing on an Illumina MiSeq platform and targeted, exon-level microarray copy number analysis.}

Results We identified causative mutations in 71/400 patients (18\%). The diagnostic rate was highest among those with seizure onset within the first two months of life (39\%), although overall it was similar in those with and without seizures. The most frequently mutated gene was SCN2A (11 patients, 3\%). Other recurrently mutated genes included CDKL5, KCNQ2, SCN8A (six patients each), FOXG1, MECP2, SCN1A, STXBP1 (five patients each), KCNT1, PCDH19, TCF4 (three patients each) and ATP1A3, PRRT2 and SLC9A6 (two patients each). Mutations in EHMT1, GABRB3, LGI1, MBD5, $P I G A, U B E 3 A$ and ZEB2 were each found in single patients. We found mutations in a number of genes in patients where either the electroclinical features or dysmorphic phenotypes were atypical for the identified gene. In only 11 cases (15\%) had the clinician sufficient certainty to specify the mutated gene as the likely cause before testing.

Conclusions Our data demonstrate the considerable utility of a gene panel approach in the diagnosis of patients with early-onset epilepsy and severe developmental delay disorders., They provide further insights into the phenotypic spectrum and genotypephenotype correlations for a number of the causative genes and emphasise the value of exon-level copy number testing in their analysis.

\section{INTRODUCTION}

Seizures affect approximately 7 in 10000 children before the age of 2 years and 1 in 500 before 5 years and are often associated with developmental delay. ${ }^{1-3}$ An increasing number of causative genes are recognised in what is emerging as an overlapping group of disorders with varying prevalence and severity of seizures, developmental delay and, in some cases, dysmorphic features or congenital malformations. ${ }^{45}$

Standard diagnostic approaches include biochemical and enzyme analysis for neurometabolic disorders, MRI brain imaging and genome-wide microarray analysis. ${ }^{1}$ Where these investigations do not identify a structural brain anomaly, biomarkers for a neurometabolic disorder, or chromosomal $\mathrm{CNV}$, diagnosis is often challenging and has traditionally been dependent on the recognition of a characteristic phenotype followed by targeted single-gene testing. Examples include SCN1A-related seizure disorders and classical Rett syndrome (MECP2). ${ }^{67}$

With increasing published literature on the wide spectrum of molecular aetiologies in these patients, targeted gene testing has allowed diagnosis in an increasing number of patients. Children with early-onset seizures and severe developmental delay (early infantile epileptic encephalopathy (EIEE)) can often be classified according to their electroclinical phenotype, for example, Ohtahara syndrome, West syndrome and epilepsy of infancy with migrating focal seizures (EIMFS). ${ }^{8}$ For some cases, accurate EIEE syndrome classification can assist in the identification of causative genes. Similarly, the recognition of a number of neurobehavioural and dysmorphic syndromes also helps identify disease-causing genes in some individuals. ${ }^{9}$ However, for many cases, clinical prediction of causative genes is challenging due to genetic heterogeneity, phenotypic pleiotropy, and for some patients, the phenotype is not sufficiently distinctive to accurately predict the causative gene. In such cases, sequential single-gene testing is timeconsuming, costly and often unsuccessful.

With the increasing availability of nextgeneration sequencing technologies, it is becoming possible to rely less on targeted single-gene analysis. This has the potential to improve diagnostic rates. It can also clarify and broaden the phenotypic spectrum of genes by reducing ascertainment bias. $^{10}$

We, therefore, chose to explore the diagnostic utility and genotype-phenotype insights provided by analysis of a panel of 46 genes in 400 patients with early-onset seizures and/or severe developmental delay. 


\section{METHODS}

\section{Patients and samples}

Four hundred patients with early-onset seizure disorders and/or severe developmental delay were referred and consented for gene panel testing by a paediatric neurologist or clinical geneticist, from a number of tertiary centres in the UK. Recruited patients did not have major structural brain malformations nor clinically significant copy number defects on microarray. Standardised clinical information was collected using a pre-test questionnaire completed by the recruiting clinician. Lymphocyte DNA was collected in all cases using standard procedures.

We selected genes for analysis in the panel that were established causes of early-onset seizures and/or severe developmental delay in patients without frequent major structural brain anomalies. Genes leading to neurometabolic disorders with readily identifiable blood/urine/cerebrospinal fluid (CSF) biomarkers were not included in the panel. The genes analysed were $A D S L$, ALG13, ARHGEF9, ARX, ATP1A3, ATRX, CDKL5, CHD2, CHRNA4, CHRNB2, CNTNAP2, EHMT1, FOXG1, GABRB3, GRIN2A, GRIN2B, KCNQ2, KCNT1, KIAA1279, LGI1, MAGI2, MBD5, MECP2, MEF2C, NRXN1, PCDH19, PIGA, PLCB1, PNKP, POLG, PRRT2, SCN1A, SCN2A, SCN8A, SLC16A2, SLC25A22, SLC2A1, SLC9A6, SPTAN1, STXBP1, SYNGAP1, TBC1D24, TCF4, UBE2A, UBE3A and ZEB2. We performed our analyses over 18 months in three phases, adding newly discovered genes to the panel as they were identified and employing improved sequence capture technology as it became available (see online supplementary table S1). In phase 1, 48 patients were analysed through 29 genes. In phase 2, 94 patients were analysed through 39 genes. In phase 3, 258 patients were analysed through 46 genes (see online supplementary table S1). Patients in phases 1 and 2 were not retested with later versions of the panel.

\section{Sequence analysis}

All patients were sequenced using a MiSeq platform (Illumina). Parental DNA was only analysed to follow-up on potentially pathogenic variants (described below). Phase 1 cases were analysed using the Haloplex sequence capture system (Agilent). Phase 2 cases were analysed using the TrueSeq Custom Amplicon (TSCA) system (Illumina), and the phase 3 cases were analysed using the SureSelectXT system (Agilent). Sequence capture libraries were designed to capture the full transcript of the target genes plus 50 bps at each intron-exon boundary using the proprietary design tools, respectively. Libraries were captured using the standard protocols for each sequence capture system and sequencing performed using standard protocols for the MiSeq platform. Barcoding allowed multiplexing of 16 cases per flow cell.

We analysed sequence data using an in-house pipeline. Regions of interest were defined in BED file format by uploading human gene nomenclature committee (HGNC) genes names to the UCSC table browser. ${ }^{11}$ Sequence reads in FASTQ format were aligned to the reference human genome (hg19) using BWA (0.6.1-r104) and default settings. ${ }^{12}$ Variant calling was performed across the entire region of interest using VarScan2 (V.2.3.7) with the following settings: minimum $30 \times$ coverage, minimum five alternate reads, minimum phred-like base-quality of $20 .^{13}{ }^{14}$ Variant calls in VCF format were then annotated using Ensembl Variant Effect Predictor (V.73) and the output parsed using an in-house script, which converts the annotated VCF file into Excel format for subsequent variant filtering and prioritisation. ${ }^{15}$ For each case, coverage was assessed across the coding exons of the target genes and their intron-exon boundaries ( $+6 \mathrm{bp}$ and $-12 \mathrm{bp}$ ) and expressed as the percentage of bases covered at $\geq 30 \times$.

We used Sanger sequencing to analyse the commonly mutated GC-rich amplicon of the $A R X$ gene that was not well targeted by our next-generation assays.

We also Sanger sequenced custom-designed amplicons to confirm potentially disease-causing variants in probands and perform segregation analysis in parental lymphocyte DNA samples. We performed PCR using MegaMix (Microzone, UK), purified PCR products using AmpureXP (Beckman Coulter, UK) and sequenced them using Big Dye Terminator V.3.1 Cycle Sequencing Kit (Applied Biosystems, USA). We used Mutation Surveyor (SoftGenetics, USA) to align traces to the reference sequence and call variants. Variant prioritisation and reporting was performed as a joint clinical laboratory process to optimise interpretation of both molecular and clinical data.

\section{Copy number analysis}

In 33 mutation negative cases from phase 1, we performed exon-level copy number analysis using a custom Roche Nimblegen oligonucleotide $135 \mathrm{~K}$ aCGH (see online supplementary table S1). Arrays were designed using proprietary Nimblegen software and read using a Roche Nimblegen MS 200 scanner. We analysed array data using CGH Fusion (infoQuant). We confirmed copy number abnormalities and performed segregation analysis in parental lymphocyte DNA samples using multiplex ligation-dependent probe amplification (MLPA) and/or FISH.

\section{RESULTS}

We identified causative mutations in 71 patients $(18 \%$; see table 1 , online supplementary table S1 and figure 1$)$. The most frequently mutated gene was $S C N 2 A$ (11 patients, 3\%), occurring de novo in all patients. Other recurrently mutated genes included CDKL5, KCNQ2, SCN8A (six patients each), FOXG1, MECP2, SCN1A, STXBP1 (five patients each), KCNT1, PCDH19, TCF4 (three patients each) and ATP1A3, PRRT2 and SCL9A6 (two patients each). Mutations in EHMT1, GABRB3, LGI1, MBD5, PIGA, $U B E 3 A$ and $Z E B 2$ were each found in single patients. Of the 54 patients for whom parental samples were available, 51 mutations were confirmed as de novo. For two patients, both harbouring KCNQ2 variants, mutations were inherited from a similarly affected parent. One FOXG1 variant had been inherited from the child's mother, who was mosaic for the mutation and clinically unaffected. Sixty-eight variants were either point mutations or small insertions and deletions. Three were larger de novo copy number abnormalities detected on our targeted array, a deletion encompassing MBD5 and two other genes (confirmed by FISH using probe RP11-548K3), a single-exon deletion within MECP2

Table 1 Summary of the 400 patients analysed

\begin{tabular}{llll}
\hline & Cases & $\begin{array}{l}\text { Cases with } \\
\text { mutation }\end{array}$ & $\begin{array}{l}\text { Percentage } \\
\text { with mutation (\%) }\end{array}$ \\
\hline All cases & 400 & 71 & 18 \\
Male & 192 & 41 & 21 \\
Female & 208 & 29 & 14 \\
Seizures & 323 & 60 & 19 \\
$\quad$ Seizure onset $<2$ years & 222 & 31 & 14 \\
$\quad$ Seizure onset $<2$ months & 77 & 30 & 39 \\
Developmental delay, no seizures & 77 & 11 & 14 \\
\hline
\end{tabular}




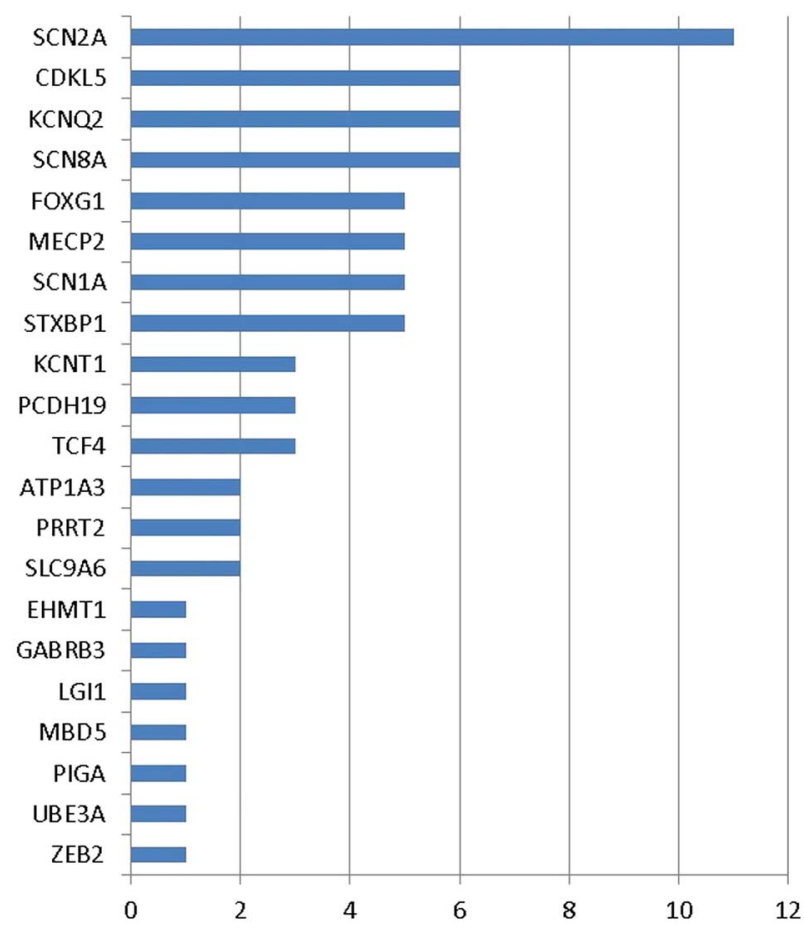

Figure 1 Number of cases with mutations in each gene.

and a multiexon duplication within CDKL5 (both confirmed by MLPA using kits P015-E1 and P189-B1, respectively).

Mutations were identified in patients with a broad range of phenotypes including EIEE syndromes, Dravet syndrome, neonatal seizures and a range of severe developmental delay phenotypes without seizures or with infrequent or resolved seizures (see tables 1 and 2 and online supplementary table S2). Mutations were identified in 60 of the $323(18 \%)$ individuals with seizures, including 30 of the $76(39 \%)$ with seizure onset under 2 months of age. Mutations were identified in 11 of the 77 (14\%) without seizures.

Mean coverage varied substantially between platforms, with a mean of $90.0 \%$ bases covered at $\geq 30 \times$ for samples analysed using the Haloplex system, $85.0 \%$ bases for the TSCA system and $99.8 \%$ for the SureSelect system.

\section{DISCUSSION}

Our panel detected clearly pathogenic mutations in $18 \%$ of our cases, demonstrating its significant diagnostic utility in patients with early-onset seizure disorders and/or severe developmental delay. This diagnostic rate is impressive given the large number of previous investigations that many of the cases had undergone. In many cases, this had included numerous sequential singlegene testing.

Mutations were found in patients with a broad range of phenotypes (tables 1 and 2). The mutation detection rate was similar in those with seizures (18\%) and those without seizures (14\%). The mutation detection rate was highest among those with early-onset seizures, before the age of 2 months (39\%).

In only 11 cases $(15 \%)$ had the clinician sufficient clinical certainty to specify on the pre-test questionnaire the actual mutated gene as the likely cause before genetic testing. These included one patient with an ATP1A3 mutation, presenting with seizures, developmental delay and alternating hemiplegia (case 1); two with inherited KCNQ2 mutations, both presenting with autosomal-dominant neonatal seizures (cases 20 and 21); one with a KCNT1 mutation presenting with EIMFS (case 24); three with MECP2 mutations presenting with classical Rett syndrome (cases 27, 28 and 30); one with a PRRT2 mutation presenting with kinesogenic dyskinesia (case 36); one with an SCN1A mutation presenting with Dravet syndrome (case 39); one with a TCF4 mutation presenting with dysmorphism characteristic of Pitt-Hopkins syndrome (case 68); and one with a ZEB2 mutation presenting with facial dysmorphism and congenital malformations characteristic of Mowat-Wilson syndrome (case 71). However, it was clearly evident from analysis of the pre-test clinical proforma that even among these, the clinician often considered a wider range of genes as possibly causative for their patient's clinical phenotype.

In many of the remaining cases, panel analysis provided a diagnosis that would not otherwise have been reached using conventional approaches or reduced the time, number of investigations and cost to make the diagnosis. In turn, this allowed appropriate advice to be given on prognosis, the tailoring of medical management (eg, antiepileptic medication considered suitable for specific genotypes) and accurate advice on risks of recurrence for future pregnancies.

In a number of cases, electroclinical diagnoses considered strongly suggestive of the causative gene were only reached after re-examination of the phenotype in the light of the identified mutation. For example, we found a KCNT1 mutation in a child later recognised to have classical EIMFS (case 23) rather than the initial diagnosis of epileptic encephalopathy with multifocal EEG discharges.

Similarly, in other cases, identification of the causative mutation allowed retrospective identification of a dysmorphic syndrome that is often considered recognisable. We identified MECP2 mutations in two children whose clinical features were consistent with classical Rett syndrome but in whom it had not been considered the most likely causative gene (cases 29 and 31). We identified TCF4 mutations in two children with severe developmental delay and facial features later confirmed to be in keeping with Pitt-Hopkins syndrome (cases 67 and 69). We also reported a UBE3A mutation in a child previously tested for Angelman methylation defects but in whom a UBE3A mutation was not considered sufficiently likely to warrant single-gene mutation analysis of UBE3A (case 70).

The majority of mutations were found in cases where the phenotype was either not easily distinguishable from that caused by a number of other genes or was atypical for the previously reported phenotype(s), emphasising the benefit of a gene panel approach over targeted single-gene testing. The extent to which more detailed pre-test phenotyping could ameliorate this varies between genes. Many of the patients described in this series had undergone extensive clinical and electroclinical workup by experts in these disorders prior to panel testing, illustrating the challenges in recognising the causative gene based on phenotype.

De novo monoallelic mutations in SCN2A are an increasingly recognised cause of an early-onset seizure and developmental delay. ${ }^{16}$ Despite its identification in 2001, testing of the gene has only become widely available with the advent of nextgeneration sequencing. ${ }^{17}$ SCN $2 A$ was the most frequently mutated gene in our series, accounting for 11 of 71 mutations and $3 \%$ of the series overall (figure 1 and table 2 ). The majority of mutations were de novo missense variants and occurred in cases with EIEE and severe or profound developmental delay. The median age of onset of seizures was 1 day of age (range 1 day to 2 years 11 months). The only child with a truncating mutation in SCN2A (case 48) presented with autism and clusters 
Table 2 Summary of the 71 mutation-positive cases

\begin{tabular}{|c|c|c|c|c|c|c|}
\hline Case & Gene & Mutation & Inheritance & Gender & Diagnosis at referral for testing & Seizures \\
\hline $1^{*}$ & ATP1A3 & het c.958G>A; p.(Ala320Thr) & De novo & $\mathrm{M}$ & Possible ATP1A3-related disorder & Yes \\
\hline 2 & ATP1A3 & het c.2839G>A; p.(Gly947Arg) & Not in mother & M & Developmental delay with seizures & Yes \\
\hline 3 & $C D K L 5$ & hemi c.532C>T; p.(Arg178Trp) & De novo & $\mathrm{M}$ & EIEE & Yes \\
\hline 4 & $C D K L 5$ & hemi c.2152G>A; p.(Val718Met) & Unknown & M & EIEE & Yes \\
\hline 5 & $C D K L 5$ & hemi c.2177_2186delinsAATGTGTCAAC; p.(Ser726*) & De novo & M & EIEE & Yes \\
\hline 6 & $C D K L 5$ & het duplication exons 6-11 & De novo & $\mathrm{F}$ & EIEE & Yes \\
\hline 7 & $C D K L 5$ & het c.167_168del; p.(Thr56Asnfs*6) & De novo & $\mathrm{F}$ & EIEE & Yes \\
\hline 8 & $C D K L 5$ & het c.965del; p.(Thr322Asnfs*28) & Unknown & $\mathrm{F}$ & EIEE & Yes \\
\hline 9 & EHMT1 & het c.1596del; p.(Thr533Profs*30) & De novo & $\mathrm{M}$ & Developmental delay with seizures & Yes \\
\hline 10 & FOXG1 & het c.256dup; p.(GIn86Profs*35) & Unknown & $\mathrm{M}$ & EIEE & Yes \\
\hline 11 & FOXG1 & het c.572T>G; p.(Met191Arg) & $\begin{array}{l}\text { From mosaic } \\
\text { mother }\end{array}$ & M & Developmental delay with seizures & Yes \\
\hline 12 & FOXG1 & het c.651C>G; p.(Tyr217*) & De novo & $\mathrm{F}$ & Developmental delay & No \\
\hline 13 & FOXG1 & het c.695A>G ; p.(Asn232Ser) & De novo & $\mathrm{M}$ & Developmental delay with movement disorder & No \\
\hline 14 & FOXG1 & het c.1188C>A; p. (Cys396*) & De novo & M & Developmental delay with movement disorder & No \\
\hline 15 & GABRB3 & het c.860C>T; p.(Thr287lle) & De novo & $\mathrm{M}$ & EIEE & Yes \\
\hline 16 & KCNQ2 & het c.601C>T; p.(Arg201Cys) & Unknown & $\mathrm{F}$ & EIEE & Yes \\
\hline 17 & KCNQ2 & het c.637C>T; p.(Arg213Trp) & Unknown & $\mathrm{F}$ & EIEE & Yes \\
\hline 18 & KCNQ2 & het c.638G>A; p.(Arg213GIn) & Unknown & $\mathrm{M}$ & EIEE & Yes \\
\hline 19 & KCNQ2 & het c.1681C>T; p.(Pro561Ser) & De novo & M & EIEE & Yes \\
\hline $20 *$ & KCNQ2 & het c.1741C>T; p.(Arg581*) & From father & $\mathrm{M}$ & Neonatal seizures & Yes \\
\hline $21^{*}$ & KCNQ2 & het c.1741C>T; p.(Arg581*) & From mother & $\mathrm{M}$ & Neonatal seizures & Yes \\
\hline 22 & KCNT1 & het c.862G>A; p.(Gly288Ser) & Unknown & M & Developmental delay with seizures & Yes \\
\hline 23 & KCNT1 & het c.2687T>A; p.(Met896Lys) & De novo & $\mathrm{F}$ & EIEE & Yes \\
\hline $24^{*}$ & KCNT1 & het c.2800G>A; p.(Ala934Thr) & De novo & M & EIMFS & Yes \\
\hline 25 & $L G / 1$ & het c.1A>G p.(Met1?) & Unknown & $\mathrm{M}$ & EIEE & Yes \\
\hline 26 & MBD5 & $\begin{array}{l}\text { het del chr2:149219863-149796844 including MBD5, EPC2, } \\
\text { KIF5C }\end{array}$ & De novo & M & Developmental delay & Yes \\
\hline $27 *$ & $M E C P 2$ & het exon 4 deletion & De novo & $\mathrm{F}$ & Rett syndrome & Yes \\
\hline $28^{*}$ & MECP2 & het c.62+2_62+3del & Unknown & $\mathrm{F}$ & Possible Rett syndrome & No \\
\hline 29 & MECP2 & het $c .844 C>T ; p .\left(\operatorname{Arg} 282^{*}\right)$ & Unknown & $\mathrm{F}$ & Developmental delay with seizures & Yes \\
\hline $30 *$ & MECP2 & het c.952C>T; p.(Arg318Cys) & De novo & $\mathrm{F}$ & Rett syndrome & No \\
\hline 31 & MECP2 & het c.1119_1147del; p.(Lys375Leufs*20) & Unknown & $\mathrm{F}$ & Developmental delay with seizures & Yes \\
\hline 32 & PIGA & het c.1064T>C; p.(Leu355Ser) & De novo & M & EIEE & Yes \\
\hline 33 & PCDH19 & het c.688G>A; p.(Asp230Asn) & De novo & $\mathrm{F}$ & EIEE & Yes \\
\hline 34 & PCDH19 & het c.707C>T; p.(Pro236Leu) & De novo & $\mathrm{F}$ & EIEE & Yes \\
\hline 35 & PCDH19 & het c.1882dup; p.(Arg628Profs*12) & De novo & $\mathrm{F}$ & EIEE & Yes \\
\hline $36^{*}$ & PRRT2 & het c.649dup; p.(Arg217Profs*8) & Unknown & M & Kinesogenic dyskinesia & No \\
\hline 37 & PRRT2 & het c.1021T>C; p. $\left({ }^{*} 341\right.$ Argext*28) & Unknown & $\mathrm{F}$ & Infantile seizure disorder & Yes \\
\hline 38 & SCN1A & het c.302G>A; p.(Arg101Gln) & De novo & M & EIEE & Yes \\
\hline $39 *$ & SCN1A & het c.2589+1_2589+2dup & De novo & M & Dravet syndrome & Yes \\
\hline 40 & SCN1A & het c.3851G>A; p.(Trp1284*) & Unknown & $\mathrm{M}$ & Developmental delay with seizures & Yes \\
\hline 41 & SCN1A & het c.4034C>T; p.(Pro1345Leu) & De novo & $\mathrm{F}$ & EIMFS & Yes \\
\hline 42 & SCN1A & het c.5010_5013del; p.(Phe1671Thrfs*8) & De novo & $\mathrm{F}$ & EIEE & Yes \\
\hline 43 & SCN2A & het c.2619C>G; p.(Ile873Met) & De novo & $\mathrm{F}$ & EIEE with movement disorder & Yes \\
\hline 44 & SCN2A & het c.2960G>T; (p.Ser987ıle) & De novo & $\mathrm{F}$ & EIEE & Yes \\
\hline 45 & SCN2A & het c.2995G>A; p.(Glu999Lys) & De novo & $\mathrm{M}$ & EIEE & Yes \\
\hline 46 & SCN2A & het c.2996A>T; p.(Glu999Val) & De novo & $\mathrm{F}$ & EIEE & Yes \\
\hline 47 & SCN2A & $\begin{array}{l}\text { het c.3778A>G; p.(Lys1260Glu) and c.3778A>C; p. } \\
\text { (Lys1260Gln) mosaic }\end{array}$ & De novo & M & EIEE & Yes \\
\hline 48 & SCN2A & het c.4303C>T; p. $\left(\operatorname{Arg} 1435^{*}\right)$ & De novo & M & Autism with seizures & Yes \\
\hline 49 & SCN2A & het c.4436A>C; p.(GIn1479Pro) & De novo & $\mathrm{M}$ & EIEE & Yes \\
\hline 50 & SCN2A & het c.4949T>C; p.(Leu1650Pro) & De novo & $\mathrm{M}$ & EIEE & Yes \\
\hline 51 & SCN2A & het c.5485C>T; p.(Leu1829Phe) & De novo & $\mathrm{M}$ & EIEE & Yes \\
\hline 52 & SCN2A & het $c .5645 G>A ; p .(\operatorname{Arg} 1882 G \ln )$ & De novo & $\mathrm{M}$ & EIEE & Yes \\
\hline 53 & SCN2A & het c.5645G>A; p.(Arg1882Gln) & De novo & M & EIEE & Yes \\
\hline 54 & SCN8A & het c.1222G>A; p.(Ala408Thr) & De novo & $\mathrm{M}$ & EIEE with movement disorder & Yes \\
\hline 55 & SCN8A & het c.3943C>G; p.(Val1315Met) & De novo & $\mathrm{F}$ & EIEE & Yes \\
\hline 56 & SCN8A & het c.3967G>T; p.(Ala1323Ser) & De novo & $\mathrm{M}$ & EIEE & Yes \\
\hline
\end{tabular}


Table 2 Continued

\begin{tabular}{|c|c|c|c|c|c|c|}
\hline Case & Gene & Mutation & Inheritance & Gender & Diagnosis at referral for testing & Seizures \\
\hline 57 & SCN8A & het c.3979A>G; p.(lle1327Val) & De novo & M & EIEE & Yes \\
\hline 58 & SCN8A & het c.5261T>C; p.(Phe1754Ser) & De novo & M & EIEE & Yes \\
\hline 59 & SCN8A & het c.5594T>C; p.(Leu1865Pro) & De novo & $\mathrm{F}$ & EIEE & Yes \\
\hline 60 & SLC9A6 & hemi c.608del p.(His203Leufs*10) & De novo & M & $\begin{array}{l}\text { Developmental delay with seizures and } \\
\text { movement disorder }\end{array}$ & Yes \\
\hline 61 & SLC9A6 & hemi c.1222_1226del; p.(His408Asnfs²) & De novo & M & EIEE & Yes \\
\hline 62 & STXBP1 & het $c .37+1 \mathrm{G}>\mathrm{A}$ & De novo & $\mathrm{F}$ & EIEE & Yes \\
\hline 63 & STXBP1 & het c.842T>C; p.(Leu281Pro) & De novo & $\mathrm{F}$ & Developmental delay with seizures & Yes \\
\hline 64 & STXBP1 & het $c .875 \mathrm{G}>\mathrm{A}$; $\mathrm{p}$.(Arg292His) & De novo & M & EIEE & Yes \\
\hline 65 & STXBP1 & het c.1019_1020del; p.(Glu340Alafs*12) & Unknown & M & Neonatal seizures including infantile spasms & Yes \\
\hline 66 & STXBP1 & het $c .1249+1 G>T$ & Unknown & $\mathrm{F}$ & EIEE & Yes \\
\hline 67 & TCF4 & het c.826C>T; p. $\left(\operatorname{Arg} 276^{*}\right)$ & De novo & $\mathrm{F}$ & Developmental delay & No \\
\hline $68^{*}$ & TCF4 & het $c .1065 C>G ; p .(S e 355 A r g)$ & De novo & $\mathrm{F}$ & Pitt-Hopkins syndrome & No \\
\hline 69 & TCF4 & het $c .1296+1 G>T$ & De novo & M & Developmental delay & No \\
\hline 70 & UBE3A & het c.2572_2576dup; p.(Lys859Asnfs*7) & Unknown & $\mathrm{F}$ & Developmental delay & No \\
\hline $71^{*}$ & ZEB2 & het c.2083C>T; p. $\left(\operatorname{Arg} 695^{*}\right)$ & De novo & M & Mowat-Wilson syndrome & No \\
\hline
\end{tabular}

${ }^{*}$ Cases in which referring clinician correctly nominated the causative gene at referral for testing.

EIEE, early infantile epileptic encephalopathy; EIMFS, epilepsy of infancy with migrating focal seizures; F, female; M, male.

of seizures from 9 months of age. These observations provide further support for the proposed association of truncating mutations with a milder seizure phenotype. ${ }^{16}$ This child was one of two with only moderate developmental delay. A further child (case 47) was apparently mosaic for two different de novo missense variants at the same residue: c.3778A $>$ G; p.(Lys1260Glu) and c.3778A $>$ C; p.(Lys1260Gln). He had better seizure control than the other children with SCN2A missense mutations and a somewhat milder developmental phenotype, suggesting that one or both of the variants may be attenuating the phenotype. Case 43, a profoundly developmentally delayed 14-year-old girl with an SCN2A missense mutation, c.2619C>G; p.(Ile873Met), had developed a generalised dystonia/dyskinetic movement disorder and had an abnormal electroretinogram. These latter features have not previously been reported to our knowledge with SCN2A mutations. This child was also homozygous for the previously reported $P N K P$ variant c.58C $>\mathrm{T}$; p.(Pro20Ser). ${ }^{18}$ As discussed below, the contribution of this PNKP variant to her phenotype is uncertain.

De novo monoallelic missense mutations in SCN8A are a recently recognised cause of early-onset seizures with developmental delay. ${ }^{19}{ }^{20}$ We identified six such SCN8A mutations in our series. All patients had early onset seizures, with a median age of onset of 5 weeks of age (range 1 day to 5 months). In all cases, the electroclinical phenotype was relatively non-specific. In the five patients where we have data, the developmental profile is of severe or profound developmental delay. One child had a very good response to phenytoin. Other clinical and radiological manifestations may also be features of the condition. One child with profound developmental delay (case 54) had a spastic dystonic movement disorder aged 9 years. This child additionally had marked hypomyelination on MRI of the brain.

Monoallelic mutations in KCNQ2 are a well-established cause of early-onset seizures. Reported phenotypes vary from benign familial neonatal seizures to a progressive pharmacoresistant EIEE. $^{21}{ }^{22}$ We identified six KCNQ2 mutations. All had had seizures from the neonatal period, with a median age of onset of 5 days. Two apparently unrelated individuals with benign neonatal seizures (cases 20 and 21) had the same truncating mutation, c. $1741 \mathrm{C}>\mathrm{T}$ p. $\left(\operatorname{Arg} 581^{*}\right)$, which had been inherited from a similarly affected parent. $^{23}$ This mutation has been reported previously in a similar context. We identified four de novo missense mutations in children with EIEE, including two with mutations in the same codon, (c.637C $>\mathrm{T}$; p. (Arg213Trp) and c. $638 \mathrm{G}>\mathrm{A}$; p. (Arg213Gln). These findings add weight to previously reported genotype-phenotype observations for KCNQ2, with truncating mutations associated with the benign, inherited phenotype and missense mutations affecting key residues with the severe, sporadic phenotype. Cellular experiments indicate that these latter mutations may have a dominant negative effect on the function at a cellular level. ${ }^{22}$

De novo monoallelic mutations in KCNT1 are known to cause early-onset seizures with developmental delay, with the typical presentation of EIMFS. ${ }^{8}{ }^{24}$ We identified three missense mutations in our series. While all presented with early-onset focal seizures, in only one child (case 24) had EIMFS been considered the working electroclinical diagnosis prior to genetic testing. In a second (case 23), re-evaluation of the phenotype in the light of the mutation confirmed a diagnosis of EIMFS.

De novo monoallelic mutations in CDKL5 are a wellrecognised cause of EIEE and severe, Rett-like developmental delay. ${ }^{25}{ }^{26}$ CDKL5 was mutated in five patients in our series despite targeted diagnostic testing of the gene being available and frequently requested by referring clinicians. The gene is located on the $\mathrm{X}$ chromosome, and the majority of reports describe de novo X-linked dominant mutations in females. ${ }^{25}$ One mutation-positive girl in our series (case 6) had previously undergone diagnostic Sanger sequencing of the gene. This was not capable of detecting her de novo multiexon intragenic CDKL5 duplication identified by our exon-level microarray. Of the remaining four CDKL5 mutation-positive cases, three were male, highlighting the potential for under-recognition of this gene as a cause of disease in males. All male CDKL5 mutationpositive cases had been classified as having an EIEE, presenting with seizures including infantile spasms aged 3 weeks and had severe or profound developmental delay. Two of these had confirmed de novo mutations, one a truncation and one missense mutation. The third (case 4), from whom parental samples are not available, had a mutation previously reported in an affected female. 
De novo monoallelic mutations in FOXG1 are typically associated with early-onset seizures and severe, Rett-like developmental delay. ${ }^{27} 28$ Other typical features include agenesis or hypoplasia of the corpus callosum and a complex movement disorder with dyskinesia, dystonia and chorea. We found FOXG1 mutations in five individuals. Only two had seizures and severe developmental delay (cases 10 and 12). The other three mutation-positive cases had a strikingly milder phenotype. Two manifested with moderate developmental delay with a movement disorder but no seizures (cases 13 and 14). A third (case 11) had mild developmental delay with (well-controlled) seizures from 4 years. His mother was somatic mosaic for the same missense mutation and was clinically unaffected. The mutation was also present in the proband's two similarly affected sisters. We postulate that the phenotype of FOXG1 syndrome now includes a broader phenotypic spectrum including milder cases with prominent movement disorders. Our data may be in keeping with a genotype-phenotype correlation underlying this broadened phenotypic spectrum: of these milder cases, one had a nonsense mutation late in the gene (case 14). The other two had novel de novo missense variants (cases 11 and 13).

Hemizygous mutations in SLC9A6 cause an X-linked recessive disorder known as Christianson syndrome. ${ }^{29}$ It was previously referred to as X-linked Angelman syndrome and presents with early-onset seizures, microcephaly, ataxia, dystonic movements and evolving lower limb spasticity from the second decade. The phenotype has been relatively consistent in the 15 families reported to date. ${ }^{30}$ We identified mutations in SLC9A6 in two boys in our series. Both had typical features of the condition, with severe seizure disorders and severe or profound developmental delay and dystonic movements. However, the gene had not been considered the likely cause prior to testing in either boy. As such, our findings both support the existing description of the phenotype and emphasise the difficulty in recognising it. Although both of the mutations we identified were de novo, diagnosis of this X-linked recessive disorder can be associated with a high risk or recurrence in further sons of a carrier mother.

The large majority of mutations identified in our series were de novo (51 of 54 from whom parental samples were available). Partly, this reflects the fact that most of the genes analysed cause disease by de novo monoallelic mutations. This considerably aids analysis as rare variants of uncertain significance can usually be ruled out if they are inherited from an unaffected parent. Inherited variants pose greater challenges in interpretation, particularly in the absence of a characteristic phenotype or clear-cut prior evidence regarding the variant. Illustrative of these challenges are our findings in individuals with variants of uncertain significance in LGI1 and PNKP.

Inherited monoallelic loss-of-function mutations in LGI1 cause autosomal-dominant temporal lobe epilepsy. ${ }^{31}$ We identified an initiation codon mutation in LGI1 in one child in our series who presented with EIEE including infantile spasms and subsequently frequent myoclonus and severe developmental delay. There was no specific evidence of temporal lobe seizures and no family history suggestive of temporal lobe epilepsy. His parents declined testing for the LGI1 variant. While it is likely that the variant predisposes to temporal lobe epilepsy, we consider that it is unlikely to be the full explanation of his phenotype.

Biallelic loss-of-function mutations in PNKP cause an autosomal-recessive DNA repair disorder with a core phenotype of EIEE, developmental delay and microcephaly. Seventeen patients from nine families have been described to date. ${ }^{18} 3233$ Six disease-causing variants have been reported, three frameshift variants and three missense variants. A number of these variants have been found in multiple families and/or have occurred in trans with other clearly pathogenic variants, providing further evidence of their pathogenicity. We identified one of the reportedly pathogenic missense variants, c.58C > T; p.(Pro20Ser), in 9 of our 400 cases. Its frequent occurrence in our cohort and our review of other available data lead us to be uncertain regarding the pathogenicity of this variant.

PNKP c.58C $>\mathrm{T}$ has been reported in one previous case with EIEE without data regarding head circumference. ${ }^{18}$ We identified the variant in homozygous form in one child. This child, case 43 , also has a de novo monoallelic SCN2A mutation. She had EIEE, profound developmental delay and microcephaly, features that could be accounted for alone by the $S C N 2 A$ mutation. In eight others, from a range of ethnic backgrounds, we identified the variant in heterozygous form without a second mutation despite good quality sequence data across the gene. One of these (case 40) also had an SCN1A nonsense mutation, consistent with the presenting phenotype. We note that the variant has an allele frequency of 0.0091 on the Exome Variant Server (http://evs.gs.washington.edu/EVS/) and has an allele frequency of 0.0076 on the ExAC database (http://www.exac. broadinstitute.org). These figures are similar to that in our series (0.0125). Taken together, these data suggest caution in interpreting this variant as disease causing. It is likely that this is a benign polymorphism.

The cost of gene panel analysis in a diagnostic setting is now similar to that of Sanger sequencing a single gene. Therefore, while targeted single-gene Sanger sequencing may remain appropriate in some cases, for example, typical SCN1A-related phenotypes, EIMFS (KCNT1), classical Rett syndrome (MECP2) and some cases with other dysmorphic syndromes, our data argue for the use of panel-based analysis as the diagnostic genetic test of choice in the majority of individuals with early-onset seizure and severe developmental delay disorders.

With time, we expect that panel analysis will evolve to use data generated from sequencing larger numbers of genes, for example, the whole exome or the clinical exome (all known disease genes). ${ }^{10} 34$ Potential advantages include the ability to add newly discovered genes without the need to redesign the assay and to analyse larger panels of genes without running a separate test. However, we currently find the better sequence coverage offered by a carefully designed and curated panel outweighs these potential benefits in a diagnostic setting. We note that the mutation detection rate we report in our early-onset seizure cases is similar to that reported in studies employing whole-exome sequencing in similar cohorts. ${ }^{35}$

While next-generation sequencing is a relatively new technology, in many respects it is equally as robust as Sanger sequencing. In some respects, it may be more robust, for example, in the detection of low-level mosaic mutations. ${ }^{34}$ We identified two mutations that had been missed despite previous Sanger sequencing of the gene in question. An SCN1A mutation (case 39) had been missed due to a technical interpretation error, less likely with more bioinformatics-driven interpretation pipelines used in the analysis of next-generation sequencing data. A MECP2 mutation (c. $62+262+3$ delTG, in case 28) had also been missed because it affected the first exon of the gene, which many diagnostic laboratories have not sequenced until recently due to uncertainty about the most clinically relevant transcript. $^{36}$ 
Like Sanger sequencing, next-generation sequencing remains poor at detecting exon-level copy number abnormalities, particularly when samples are run in the relatively small batches typical of a diagnostic setting. ${ }^{34}$ We, therefore, piloted the use of an exon-level microarray targeting our gene panel in a subset of our series. In our analysis of 33 cases without mutations on panel sequencing, we identified three likely pathogenic copy number abnormalities. Exon-level copy number analysis is also valuable in individuals harbouring single mutations in autosomal-recessive genes to search for second mutations and assist in the interpretation of the significance of sequence variants. Until next-generation sequencing allows robust copy number analysis, exon-level microarray will be a useful component of gene panel analysis and we have now integrated it into our standard analysis work stream.

In conclusion, our work demonstrates the significant utility of a gene panel approach in the diagnosis of patients with early-onset epilepsy and severe developmental delay disorders, particularly the former. It also broadens the phenotypic spectrum, provides insights into the genotype-phenotype relationships of a number of the causative genes and emphasises the importance of exon-level copy number testing in their analysis.

\section{Author affiliations \\ ${ }^{1}$ North East Thames Regional Genetics Service, Great Ormond Street Hospital for Children, London, UK \\ ${ }^{2}$ Molecular Neurosciences, Developmental Neurosciences Programme, University College London Institute of Child Health, London, UK \\ ${ }^{3}$ Department of Neurology, Great Ormond Street Hospital for Children, London, UK ${ }^{4}$ Genetics and Genomic Medicine Unit, University College London Institute of Child Health, London, UK}

Acknowledgements The authors are grateful to the patients and families for their participation and to the referring clinicians for supporting the study. This study was supported by the National Institute for Health Research Biomedical Research Centre at Great Ormond Street Hospital for Children NHS Foundation Trust and University College London.

Contributors RS and MK designed and oversaw the study. NT, HB, RS, RP, DM and $\mathrm{L}$ performed laboratory analyses and variant interpretation. $C B$ designed the bioinformatic pipeline and supported analysis. RS, AM, HB, AP, EM, AN, JH and MK recruited patients and performed phenotypic analysis. RS wrote the manuscript with input from all other authors.

Competing interests MAK is funded by a Wellcome Intermediate Clinical Fellowship and has received funding from Great Ormond Street Children's Charities, Child Brain Research, FOLKS foundation, Gracious Heart Charity Foundation and the Rosetrees Trust.

Ethics approval NRES London.

Provenance and peer review Not commissioned; externally peer reviewed.

Open Access This is an Open Access article distributed in accordance with the terms of the Creative Commons Attribution (CC BY 4.0) license, which permits others to distribute, remix, adapt and build upon this work, for commercial use, provided the original work is properly cited. See: http://creativecommons.org/ licenses/by/4.0/

\section{REFERENCES}

1 Eltze CM, Chong WK, Cox T, Whitney A, Cortina-Borja M, Chin RF, Scott RC, Cross $\mathrm{JH}$. A population-based study of newly diagnosed epilepsy in infants. Epilepsia 2013;54:437-45.

2 Joint Epilepsy Council 2011. http://www.jointepilepsycouncil.org.uk/

3 Sander JW. The use of antiepileptic drugs - principles and practice. Epilepsia 2004;45(Suppl 6):28-34.

4 Tavyev Asher YJ, Scaglia F. Molecular bases and clinical spectrum of early infantile epileptic encephalopathies. Eur J Med Genet 2012;55:299-306.

5 Nava $C$, Dalle $C$, Rastetter $A$, Striano $P$, de Kovel CG, Nabbout $R$, Cancès C, Ville $D$, Brilstra EH, Gobbi G, Raffo E, Bouteiller D, Marie Y, Trouillard O, Robbiano A, Keren B, Agher D, Roze E, Lesage S, Nicolas A, Brice A, Baulac M, Vogt C, El Hajj $N$, Schneider E, Suls A, Weckhuysen S, Gormley P, Lehesjoki AE, De Jonghe P, Helbig I, Baulac S, Zara F, Koeleman BP; EuroEPINOMICS RES Consortium, Haaf T, LeGuern E, Depienne C. De novo mutations in HCN1 cause early infantile epileptic encephalopathy. Nat Genet 2014;46:640-5.
6 Scheffer IE, Berkovic SF. Generalized epilepsy with febrile seizures plus. a genetic disorder with heterogeneous clinical phenotypes. Brain 1997;120:479-90.

7 Neul JL, Kaufmann WE, Glaze DG, Christodoulou J, Clarke AJ, Bahi-Buisson N, Leonard H, Bailey ME, Schanen NC, Zappella M, Renieri A, Huppke P, Percy AK, RettSearch Consortium. Rett syndrome: revised diagnostic criteria and nomenclature. Ann Neurol 2010:68:944-50.

8 McTague A, Appleton R, Avula S, Cross JH, King MD, Jacques TS, Bhate S, Cronin A, Curran A, Desurkar A, Farrell MA, Hughes E, Jefferson R, Lascelles $K$, Livingston J, Meyer E, McLellan A, Poduri A, Scheffer IE, Spinty S, Kurian MA, Kneen R. Migrating partial seizures of infancy: expansion of the electroclinical, radiological and pathological disease spectrum. Brain 2013;136:1578-91.

9 Tan WH, Bird LM, Thibert RL, Williams CA. If not angelman, what is it? a review of angelman-like syndromes. Am J Med Genet A 2014;164A:975-92.

10 Schnekenberg RP, Németh AH. Next-generation sequencing in childhood disorders. Arch Dis Child 2014;99:284-90.

11 Karolchik D, Hinrichs AS, Furey TS, Roskin KM, Sugnet CW, Haussler D, Kent WJ. The UCSC table browser data retrieval tool. Nucleic Acids Res 2004;32 issue): D493-6.

12 Li H, Durbin R. Fast and accurate short read alignment with Burrows-Wheeler transform. Bioinformatics 2009;25:1754-60.

13 Koboldt DC, Chen K, Wylie T, Larson DE, McLellan MD, Mardis ER, Weinstock GM, Wilson RK, Ding L. VarScan: variant detection in massively parallel sequencing of individual and pooled samples. Bioinformatics 2009;25:2283-5.

14 Koboldt DC, Zhang Q, Larson DE, Shen D, McLellan MD, Lin L, Miller CA, Mardis ER, Ding L, Wilson RK. VarScan 2: Somatic mutation and copy number alteration discovery in cancer by exome sequencing. Genome Res 2012;22:568-76.

15 McLaren W, Pritchard B, Rios D, Chen Y, Flicek P, Cunningham F. Deriving the consequences of genomic variants with the Ensembl API and SNP Effect Predictor. Bioinformatics 2010;26:2069-70.

16 Shi X, Yasumoto S, Kurahashi H, Nakagawa E, Fukasawa T, Uchiya S, Hirose S. Clinical spectrum of SCN2A mutations. Brain Dev 2012;34:541-5.

17 Sugawara T, Tsurubuchi Y, Agarwala KL, Ito M, Fukuma G, Mazaki-Miyazaki E, Nagafuji H, Noda M, Imoto K, Wada K, Mitsudome A, Kaneko S, Montal M, Nagata K, Hirose S, Yamakawa K. A missense mutation of the Na+ channel $\alpha$ II subunit gene Nav1.2 in a patient with febrile and afebrile seizures causes channel dysfunction. Proc Natl Acad Sci USA 2001;98:6381-9.

18 Carvill GL, Heavin SB, Yendle SC, McMahon JM, O'Roak BJ, Cook J, Khan A Dorschner MO, Weaver M, Calvert S, Malone S, Wallace G, Stanley T, Bye AM, Bleasel A, Howell KB, Kivity S, Mackay MT, Rodriguez-Casero V, Webster R, Korczyn A, Afawi Z, Zelnick N, Lerman-Sagie T, Lev D, Møller RS, Gill D, Andrade DM, Freeman JL, Sadleir LG, Shendure J, Berkovic SF, Scheffer IE, Mefford HC. Targeted resequencing in epileptic encephalopathies identifies de novo mutations in CHD2 and SYNGAP1. Nat Genet 2013;45:825-30.

19 Veeramah K R, O'Brien JE, Meisler MH, Cheng X, Dib-Hajj SD, Waxman SG, Talwar $D$, Girirajan S, Eichler EE, Restifo LL, Erickson RP, Hammer MF. De novo pathogenic SCN8A mutation identified by whole-genome sequencing of a family quartet affected by infantile epileptic encephalopathy and SUDEP. Am J Hum Genet 2012;90:502-12

20 Larsen J, Carvill GL, Gardella E, Kluger G, Schmiedel G, Barisic N, Depienne C, Brilstra E, Mang Y, Nielsen JE, Kirkpatrick M, Goudie D, Goldman R, Jähn JA, Jepsen B, Gill D, Döcker M, Biskup S, McMahon JM, Koeleman B, Harris M, Braun $\mathrm{K}$, de Kovel CG, Marini C, Specchio N, Djémié T, Weckhuysen $S$, Tommerup N, Troncoso M, Troncoso L, Bevot A, Wolff M, Hjalgrim H, Guerrini R, Scheffer IE, Mefford HC, Møller RS, EuroEPINOMICS RES Consortium CRP. The phenotypic spectrum of SCN8A encephalopathy. Neurology 2015;84:480-9.

21 Singh N A, Charlier C, Stauffer D, DuPont B R, Leach R J, Melis R, Ronen GM, Bjerre I, Quattlebaum T, Murphy JV, McHarg ML, Gagnon D, Rosales TO, Peiffer A, Anderson VE, Leppert M. A novel potassium channel gene, KCNQ2, is mutated in an inherited epilepsy of newborns. Nat Genet 1998;18:25-9.

22 Miceli F, Soldovieri MV, Ambrosino P, Barrese V, Migliore M, Cilio MR, Taglialatela $M$. Genotype-phenotype correlations in neonatal epilepsies caused by mutations in the voltage sensor of Kv7.2 potassium channel subunits. Proc Natl Acad Sci USA 2013;110:4386-91.

23 Singh NA, Westenskow P, Charlier C, Pappas C, Leslie J, Dillon J, Anderson VE, Sanguinetti MC, Leppert MF; BFNC Physician Consortium. KCNQ2 and KCNQ3 potassium channel genes in benign familial neonatal convulsions: expansion of the functional and mutation spectrum. Brain 2003;126:2726-37.

24 Barcia G, Fleming MR, Deligniere A, Gazula VR, Brown MR, Langouet M, Chen $H$, Kronengold J, Abhyankar A, Cilio R, Nitschke P, Kaminska A, Boddaert N, Casanova JL, Desguerre I, Munnich A, Dulac O, Kaczmarek LK, Colleaux L, Nabbout R. De novo gain-of-function KCNT1 channel mutations cause malignant migrating partial seizures of infancy. Nat Genet 2012:44:1255-9.

25 Fehr S, Wilson M, Downs J, Williams S, Murgia A, Sartori S, Vecchi M, Ho G, Polli $\mathrm{R}$, Psoni S, Bao X, de Klerk N, Leonard H, Christodoulou J. The CDKL5 disorder is an independent clinical entity associated with early-onset encephalopathy. Eur J Hum Genet 2013;21:266-73.

26 Tao J, Van Esch H, Hagedorn-Greiwe M, Hoffmann K, Moser B, Raynaud M, Sperner J, Fryns JP, Schwinger E, Gécz J, Ropers HH, Kalscheuer VM. Mutations in 
the X-linked cyclin-dependent kinase-like 5 (CDKL5/STK9) gene are associated with severe neurodevelopmental retardation. Am J Hum Genet 2004;75:1149-54.

27 Ariani F, Hayek G, Rondinella D, Artuso R, Mencarelli MA, Spanhol-Rosseto A, Pollazzon M, Buoni S, Spiga O, Ricciardi S, Meloni I, Longo I, Mari F, Broccoli V, Zappella M, Renieri A. FOXG1 is responsible for the congenital variant of Rett syndrome. Am J Hum Genet 2008;83:89-93.

28 Kortüm F, Das S, Flindt M, Morris-Rosendahl DJ, Stefanova I, Goldstein A, Horn D, Klopocki E, Kluger G, Martin P, Rauch A, Roumer A, Saitta S, Walsh LE, Wieczorek $D$, Uyanik G, Kutsche K, Dobyns WB. The core FOXG1 syndrome phenotype consists of postnatal microcephaly, severe mental retardation, absent language, dyskinesia, and corpus callosum hypogenesis. J Med Genet 2011;48:396-406.

29 Gilfillan GD, Selmer KK, Roxrud I, Smith R, Kyllerman M, Eiklid K, Kroken M, Mattingsdal M, Egeland T, Stenmark H, Sjøholm H, Server A, Samuelsson L, Christianson A, Tarpey P, Whibley A, Stratton MR, Futreal PA, Teague J, Edkins S, Gecz J, Turner G, Raymond FL, Schwartz C, Stevenson RE, Undlien DE, Strømme P. SLC9A6 mutations cause $X$-linked mental retardation, microcephaly, epilepsy, and ataxia, a phenotype mimicking Angelman syndrome. Am J Hum Genet 2008;82:1003-10.

30 Pescosolido MF, Stein DM, Schmidt M, El Achkar CM, Sabbagh M, Rogg JM, Tantravahi U, McLean RL, Liu JS, Poduri A, Morrow EM. Genetic and phenotypic diversity of NHE6 mutations in Christianson syndrome. Ann Neurol 2014;76:581-93.
31 Kalachikov S, Evgrafov O, Ross B, Winawer M, Barker-Cummings C, Martinelli Boneschi F, Choi C, Morozov P, Das K, Teplitskaya E, Yu A, Cayanis E, Penchaszadeh G, Kottmann AH, Pedley TA, Hauser WA, Ottman R, Gilliam TC. Mutations in LGI1 cause autosomal-dominant partial epilepsy with auditory features. Nature Genet 2002;30:335-41.

32 Shen J, Gilmore EC, Marshall CA, Haddadin M, Reynolds JJ, Eyaid W, Bodell A, Barry B, Gleason D, Allen K, Ganesh VS, Chang BS, Grix A, Hill RS, Topcu M, Caldecott KW, Barkovich AJ, Walsh CA. Mutations in PNKP cause microcephaly, seizures and defects in DNA repair. Nat Genet 2010;42:245-9.

33 Poulton C, Oegema R, Heijsman D, Hoogeboom J, Schot R, Stroink H, Willemsen MA, Verheijen FW, van de Spek P, Kremer A, Mancini GM. Progressive cerebellar atrophy and polyneuropathy: expanding the spectrum of PNKP mutations. Neurogenetics 2013;14:43-51.

34 Danielsson K, Mun LJ, Lordemann A, Mao J, Lin CH. Next-generation sequencing applied to rare diseases genomics. Expert Rev Mol Diagn 2014;14:469-87.

35 Scheffer IE. Epilepsy genetics revolutionizes clinical practice. Neuropediatrics 2014;45:70-4.

36 Kriaucionis $\mathrm{S}$, Bird $\mathrm{A}$. The major form of $\mathrm{MeCP} 2$ has a novel $\mathrm{N}$-terminus generated by alternative splicing. Nucleic Acids Res 2004;32:1818-23. 